\title{
ASSESSMENT OF PERCEIVED WORK ABILITY AND ITS DETERMINANTS AMONG HEALTHCARE PROVIDERS
}

\author{
By \\ Abdelrehim MG , Mahfouz EM and Abd El Latief OK \\ Department of Public Health, Preventive and Occcupational Medicine, Faculty \\ of Medicine, Minia University, Egypt \\ Corresponding author: Abdelrehim MG: email:marwa.gamal@mu.edu.eg
}

\begin{abstract}
Introduction: Healthcare providers are at high risk of occupational stress, burnout, health hazards and job dissatisfaction which can have harmful consequences on their health, work ability and professional efficiency. Therefore, it is important to adopt strategies for the promotion of healthy working conditions and improvement of their work ability. Aim of work: To assess the perceived work ability and its dimensions among the healthcare providers at Minia city, Egypt and identifies its relationship with socio-demographics, work-related factors and chronic diseases. Materials and methods: In a cross-sectional study among 233 healthcare providers, data about sociodemographics, history of chronic diseases, work-related factors and the Work Ability Index (WAI) questionnaire were collected during an interview with the participants. Multinomial logistic regression analyses were performed to identify the determinants of WAI. Results: The study included 145 (62.2\%) nurses, 46 (19.8\%) physicians and $42(18 \%)$ technicians. The mean WAI score was $40.6( \pm 4.6)$ and the participants were categorized based on their work ability into poor (14.1\%), suboptimal (58.4\%) and optimal (27.5\%). There were significant differences in the WAI scores and most dimensions of work ability between different age groups. In the final multivariable analysis: age, sex, work category and chronic disorders (including musculoskeletal, cardiovascular, respiratory and gastrointestinal) were the significant predictors of work ability among the studied healthcare providers. Conclusion: Low work ability among health care providers was significantly associated with age, sex, work category, and chronic disease conditions. Therefore, promoting health, managing and preventing chronic diseases particularly at old age is essential in designing effective interventions to maintain and improve the work ability of healthcare providers.
\end{abstract}

Keywords: Healthcare providers, Work ability, Work ability index determinants, .Chronic diseases and Minia 


\section{Introduction}

Perceived work ability was defined as the functional capacity of the worker in a current job with consideration to the challenges or demands of the job and his available resources. The work ability is to achieve a balance between the personal resources and the job requirements and is considered a product of both the individual and the work environment (Gould et al., 2008). The work ability index (WAI) questionnaire is the most popular diagnostic tool of work ability that was developed by the Finnish Institute of Occupational Health (FIOH) to help professionals in workers' health promotion to evaluate their working capacity and define interventions aiming to restore, maintain and improve their work ability (Tuomi et al., 1998) . WAI considers the workers' self-assessed work ability in_relation to work demands, health status and a particular type of work. It has proved to be a simple, efficient and reliable method to assess work ability across different cultures and working conditions (Gould et al., 2008). It also can predict prolonged sickness absence (Guo et al., 2015 and Palmlof et al., 2019), early retirement and even worker decease (Tuomi et al., 1997 and Guo et al., 2015).
Healthcare providers face high risk of work stress, burnout, health hazards and job dissatisfaction which can have harmful consequences on their health, work ability and professional efficiency (Costa et al., 2005). The negative impact of work environment on the healthcare providers' physical, mental and emotional health can be due to the nature of the profession that imply time pressure, shift work, inappropriate working conditions, excessive workload, prolonged working hours, interpersonal conflicts and maladministration (Koinis et al., 2015). There is an apparent need for employees in the healthcare sector to remain productive until retirement age. The healthcare system in Egypt faces multiple challenges such as shortages in healthcare resources, the organizational structure and the system management need reform and the distribution and quality of human resources need to be improved (Elden et al., 2016). Although work ability research is successful in many countries around the world, little research has been conducted in Egypt, where the work conditions, poor healthcare resources, public policies for retirement are quite different from policies in other countries. Therefore, it is important to evaluate the work ability level and identify determinant factors 
that can predict the ability to work among healthcare providers. Such research can provide valuable information to the individuals as well as the organizations to identify ways to maintain and improve the work ability and mitigate premature workforce departure.

\section{Aim of work}

To assess the perceived work ability and its dimensions among the healthcare providers at Minia city, Egypt and identifies its relationship with sociodemographics, work-related factors and chronic diseases.

\section{Materials and methods}

Study design: A hospital-based cross-sectional study.

\section{Place and duration of the study:} The study was conducted among the healthcare providers of three Ministry of Health Hospitals at Minia city during the period from September to December 2019.

Study sample: The sample size was calculated using Epi Info ${ }^{\mathrm{TM}} 7$ software; and at a confidence level of $95 \%$ and power was kept $80 \%$ (prevalence of poor work ability was $20 \%$ and population were 1250), the required sample was 206 which was increased to 250 to compensate for lack of response. A stratified random sample was recruited. The health care providers in the three hospitals were divided into three strata; nurses, physicians and technicians. The total sample size was divided by the weight of subjects in each stratum and a random sample was chosen from each stratum. The study participants included those who were employed for more than one year and not on vacation during the study period. The total number of participants included in the study was 233 (145 (62.2\%) nurses, 46 (19.8\%) physicians, and 42 (18\%) technicians) with the response rate $93 \%$. Nonparticipation was mostly due to a lack of time and a high workload.

\section{Study methods:}

\section{1- Questionnaire:}

Data were collected during an interview with the healthcare providers and all information related to the study was explained to the participants before completion of the questionnaires which included:

1. Sociodemographic data, reproductive history, smoking history, and work characteristics

\section{Measurement of weight and} height to calculate Body Mass Index (BMI) as person's weight in kilograms divided by the height in meters squared. 
3. Perceived work ability was evaluated by the Work Ability Index (WAI) questionnaire in English which was completed by the investigators during an interview with the healthcare providers. The WAI questionnaire is a valid, reliable, and cross-national instrument for use in occupational health (de Zwart et al., 2002). It consists of 7 dimensions: (1) Current work ability compared with the lifetime best (scored 0-10). (2) Subjective current work ability as regards to the physical and mental demands of work (two questions each on a 5-point scale). (3) The number of diseases diagnosed by a physician (scores ranged from $1=>5$ diseases to $7=0$ diseases). (4) Estimated work impairment due to diseases (sixpoint scale ranged from 1-6). (5) Sick leave in the past 12 months (scores ranged from $5=0$ days to $1=100$ days or more). (6) Personal prognosis of work ability in the next two years (score of $1=$ hardly able to work, 4=not sure, or 7=fairly sure). (7) Mental resources in the past few months was assessed through three questions about enjoying daily activities, being active and alert, and feeling to be full of hope about the future. The answers were ranging from never $=0$ to always $=4$. The total WAI score ranges from 7- 49 and was calculated as the sum score of the 7 dimensions. Based on the WAI score the current study categorized the participants' work ability into three categories: poor $(<37)$, suboptimal (37-43) and optimal (44-49) (Tuomi et al., 1998).

\section{Consent}

The study personnel provided a verbal informed consent to participate in the study. Confidentiality and anonymity of the participants were strictly maintained through a code number on the questionnaire.

\section{Ethical approval}

The study was approved by the Ethical Committee of Faculty of Medicine, Minia University. Moreover, approvals from the Ministry of Health at Minia city to conduct the study were obtained

\section{Data management}

Statistical analyses were performed on IBM SPSS software version 25. Categorical variables were presented as frequency (No) and percentage and numerical variables were presented as mean and standard deviation (SD). Chi-square test, One-way ANOVA and Independent T-Test were used to compare different characteristics among the study participants. The p-value $<0.05$ was considered to be statistically significant. Multinomial Logistic Regression models were used to estimate the OR and $95 \% \mathrm{CI}$ for the association between the variables and perceived work ability. The work 
ability was the outcome variable and and optimal and the reference category had three categories poor, suboptimal was the suboptimal work ability.

\section{Results}

Table 1: Socio-demographics and reproductive histories of the studied healthcare providers by age group.

\begin{tabular}{|c|c|c|c|c|c|}
\hline & \multicolumn{3}{|c|}{ Age groups (years) } & \multirow[b]{2}{*}{$\begin{array}{c}\text { Total } \\
\text { No }=\mathbf{2 3 3}\end{array}$} & \multirow[b]{2}{*}{ p-value } \\
\hline & $\begin{array}{c}20-29 \\
\text { No }=121\end{array}$ & $\begin{array}{c}30-39 \\
\text { No }=64\end{array}$ & $\begin{array}{c}\geq 40 \\
\text { No }=48\end{array}$ & & \\
\hline \multicolumn{6}{|c|}{ Socio-demographic characteristics } \\
\hline \multicolumn{6}{|l|}{ Sex } \\
\hline Male & $33(27.3 \%)$ & $18(28.1 \%)$ & $14(29.2 \%)$ & $65(27.9 \%)$ & \\
\hline Female & $88(72.7 \%)$ & $46(71.9 \%)$ & $34(70.8 \%)$ & $168(72.1 \%)$ & 0.969 \\
\hline \multicolumn{6}{|l|}{ Marital status } \\
\hline Unmarried & $62(51.2 \%)$ & $12(18.8 \%)$ & $10(20.8 \%)$ & $84(36.1 \%)$ & \\
\hline Married & $59(48.8 \%)$ & $52(81.2 \%)$ & $38(79.2 \%)$ & $149(63.9 \%)$ & $<0.0001 * *$ \\
\hline \multicolumn{6}{|l|}{ Residence } \\
\hline Urban & $46(38 \%)$ & $39(60.9 \%)$ & $33(68.8 \%)$ & $118(50.6 \%)$ & \\
\hline Rural & $75(62 \%)$ & $25(39.1 \%)$ & $15(31.3 \%)$ & $115(49.4 \%)$ & $<0.0001 * *$ \\
\hline \multicolumn{6}{|l|}{ Smoking } \\
\hline Non-smoker & $88(72.7 \%)$ & $45(70.3 \%)$ & $30(62.5 \%)$ & $163(70 \%)$ & \\
\hline Smoker & $19(15.7 \%)$ & $14(21.9 \%)$ & $10(20.8 \%)$ & $43(18.5 \%)$ & 0.490 \\
\hline Ex-smoker & $14(11.6 \%)$ & $5(7.8 \%)$ & $8(16.7 \%)$ & $27(11.6 \%)$ & \\
\hline \multicolumn{6}{|l|}{ BMI a } \\
\hline $18.5-24.9$ & $63(52.1 \%)$ & $15(23.4 \%)$ & $7(14.6 \%)$ & $85(36.5 \%)$ & \\
\hline $25-29.9$ & $38(31.4 \%)$ & $26(40.6 \%)$ & $19(39.6 \%)$ & $83(35.6 \%)$ & $<0.0001 * *$ \\
\hline$\geq 30$ & $20(16.5 \%)$ & $23(36 \%)$ & $22(45.8 \%)$ & $65(27.9 \%)$ & \\
\hline \multicolumn{6}{|c|}{$\begin{array}{l}\text { Reproductive history for females }(\mathrm{No}=168) \\
\text { Menopausal status }\end{array}$} \\
\hline Premenopausal & $87(98.9 \%)$ & $41(89.1 \%)$ & $20(58.8 \%)$ & $148(88.1 \%)$ & \\
\hline Postmenopausal & $0(0 \%)$ & $0(0 \%)$ & $4(11.8 \%)$ & $4(2.4 \%)$ & $<00001 \% *$ \\
\hline Irregular menstruation & $1(1.1 \%)$ & $5(10.9 \%)$ & $10(29.4 \%)$ & $16(9.5 \%)$ & $<0.0001$ \\
\hline Number of children ${ }^{b}$ & $0.66 \pm 0.94$ & $2.40 \pm 1.10$ & $2.87 \pm 1.31$ & $1.62 \pm 1.45$ & $<0.0001 * *$ \\
\hline \multicolumn{6}{|l|}{ Breastfeeding } \\
\hline Yes & $21(23.9 \%)$ & $7(15.2 \%)$ & $2(5.9 \%)$ & $30(17.9 \%)$ & \\
\hline NO & $67(76.1 \%)$ & $39(84.8 \%)$ & $32(94.1 \%)$ & $138(82.1 \%)$ & 0.058 \\
\hline \multicolumn{6}{|c|}{ Hormonal contraception } \\
\hline Yes & $14(15.9 \%)$ & $19(41.3 \%)$ & $10(29.4 \%)$ & $43(25.6 \%)$ & \\
\hline $\mathrm{NO}$ & $74(84.1 \%)$ & $27(58.7 \%)$ & $24(70.6 \%)$ & $125(74.4 \%)$ & $0.005 *$ \\
\hline
\end{tabular}

a: BMI: Body mass index

$\mathrm{b}$ Mean \pm SD

*: Statistically significant,

**: Highly statistically significant. 
Table 1 showed that the study population included 65 males $(27.9 \%)$ and 168 females $(72.1 \%)$. The mean age of the participants was $31.8( \pm 8.9)$ years. The majority of the studied group was from $20-29$ years old $(51.9 \%), 63.9 \%$ were married, $50.6 \%$ were from urban areas, $70 \%$ were non-smokers, and $27.9 \%$ were obese personnel (BMI $\geq 30)$. The majority of female participants were neither breast feeding $(82.1 \%)$ nor taking hormonal contraception $(74.4 \%)$ at the study time.

Table 2: Chronic diseases and work characteristics of the studied healthcare providers by age group.

\begin{tabular}{|c|c|c|c|c|c|}
\hline & & ge groups (year & & & \\
\hline & $\begin{array}{c}20-29 \\
\text { No }=121\end{array}$ & $\begin{array}{c}30-39 \\
\text { No }=64\end{array}$ & $\begin{array}{c}\geq 40 \\
\mathrm{No}=48\end{array}$ & $\begin{array}{c}\text { Total } \\
\text { No }=\mathbf{2 3 3}\end{array}$ & p-value \\
\hline History of chron & eases & & & & \\
\hline $\begin{array}{l}\text { Musculoskeletal } \\
\text { NO } \\
\text { Yes }\end{array}$ & $\begin{array}{l}98(81 \%) \\
23(19 \%)\end{array}$ & $\begin{array}{l}41(64.1 \%) \\
23(35.9 \%)\end{array}$ & $\begin{array}{l}33(68.8 \%) \\
15(31.3 \%)\end{array}$ & $\begin{array}{c}172(73.8 \%) \\
61(26.2 \%)\end{array}$ & $0.030 *$ \\
\hline $\begin{array}{l}\text { Cardiovascular } \\
\text { NO } \\
\text { Yes }\end{array}$ & $\begin{array}{c}108(89.3 \%) \\
13(10.7 \%)\end{array}$ & $\begin{array}{l}47(73.4 \%) \\
17(26.6 \%)\end{array}$ & $\begin{array}{l}32(66.7 \%) \\
16(33.3 \%)\end{array}$ & $\begin{array}{c}187(80.3 \%) \\
46(19.7 \%)\end{array}$ & $0.001 *$ \\
\hline $\begin{array}{l}\text { Respiratory } \\
\text { NO } \\
\text { Yes }\end{array}$ & $\begin{array}{l}90(74.4 \%) \\
31(25.6 \%)\end{array}$ & $\begin{array}{l}45(70.3 \%) \\
19(29.7 \%)\end{array}$ & $\begin{array}{l}34(70.8 \%) \\
14(29.2 \%)\end{array}$ & $\begin{array}{c}169(72.5 \%) \\
64(27.5 \%)\end{array}$ & 0.804 \\
\hline $\begin{array}{l}\text { Gastrointestinal } \\
\text { NO } \\
\text { Yes }\end{array}$ & $\begin{array}{c}106(87.6 \%) \\
15(12.4 \%)\end{array}$ & $\begin{array}{l}51(79.7 \%) \\
13(20.3 \%)\end{array}$ & $\begin{array}{l}37(77.1 \%) \\
11(22.9 \%)\end{array}$ & $\begin{array}{c}194(83.3 \%) \\
39(16.7 \%)\end{array}$ & 0.171 \\
\hline $\begin{array}{l}\text { Mental } \\
\mathrm{NO} \\
\mathrm{Yes}\end{array}$ & $\begin{array}{c}112(92.6 \%) \\
9(7.4 \%)\end{array}$ & $\begin{array}{c}61(95.3 \%) \\
3(4.7 \%)\end{array}$ & $\begin{array}{c}43(89.6 \%) \\
5(10.4 \%)\end{array}$ & $\begin{array}{c}216(92.7 \%) \\
17(7.3 \%)\end{array}$ & 0.521 \\
\hline Work-related fac & & & & & \\
\hline $\begin{array}{l}\text { Work category } \\
\text { Nurses } \\
\text { Physicians } \\
\text { Technicians }\end{array}$ & $\begin{array}{l}84(69.4 \%) \\
18(14.9 \%) \\
19(15.7 \%)\end{array}$ & $\begin{array}{l}33(51.6 \%) \\
18(28.1 \%) \\
13(20.3 \%)\end{array}$ & $\begin{array}{l}28(58.3 \%) \\
10(20.8 \%) \\
10(20.8 \%)\end{array}$ & $\begin{array}{c}145(62.2 \%) \\
46(19.8 \%) \\
42(18 \%)\end{array}$ & 0.146 \\
\hline $\begin{array}{l}\text { Night shift } \\
\text { NO } \\
\text { Yes }\end{array}$ & $\begin{array}{l}71(58.7 \%) \\
50(41.3 \%)\end{array}$ & $\begin{array}{l}37(57.8 \%) \\
27(42.2 \%)\end{array}$ & $\begin{array}{l}28(58.3 \%) \\
20(41.7 \%)\end{array}$ & $\begin{array}{c}136(58.4 \%) \\
97(41.6 \%)\end{array}$ & 0.994 \\
\hline
\end{tabular}


Specialty

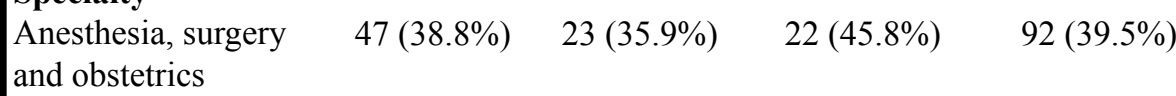

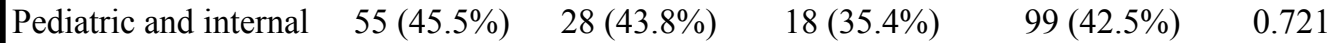

medicine

Laboratory

technicians

Working hours per day ${ }^{\text {a }}$

$19(15.7 \%) \quad 13(20.3 \%) \quad 10(20.8 \%) \quad 42(18 \%)$

$\begin{array}{lllll}7.77 \pm 1.91 & 7.62 \pm 2.24 & 7.87 \pm 2.40 & 7.75 \pm 2.11 & 0.812\end{array}$

$\begin{array}{llllll}\text { Time to reach work } & 36.50 \pm 22.35 & 34.87 \pm 22.88 & 34.17 \pm 24.19 & 35.55 \pm 22.81 & 0.814\end{array}$ (in minutes) ${ }^{a}$

a :Mean \pm SD

*: Statistically significant,

**: Highly statistically significant.

Table 2 showed that old participants ( $\geq 40$ years) were more likely to have cardiovascular diseases than younger ones (statistically significant) also musculoskeletal disorders were statistically significantly higher among the personnel with age group from 30-39years old.

Table 3: The Work Ability Index (WAI) and its dimensions among the studied health care providers by age group.

\begin{tabular}{|c|c|c|c|c|c|}
\hline \multirow[b]{2}{*}{ WAI score and dimensions } & \multicolumn{3}{|c|}{ Age groups (years) } & \multirow[b]{2}{*}{$\begin{array}{c}\text { Total } \\
\text { No }=233\end{array}$} & \multirow[b]{2}{*}{ p-value } \\
\hline & $\begin{array}{c}20-29 \\
\mathrm{No}=121\end{array}$ & $\begin{array}{c}\text { 30-39 } \\
\mathrm{No}=64\end{array}$ & $\begin{array}{c}\geq 40 \\
\text { No }=48\end{array}$ & & \\
\hline \multicolumn{6}{|l|}{ WAI score } \\
\hline $\begin{array}{l}\text {-Poor } \\
\text {-Suboptimal } \\
\text {-Optimal }\end{array}$ & $\begin{array}{c}13(10.8 \%) \\
62(51.2 \%) \\
46(38 \%)\end{array}$ & $\begin{array}{c}8(12.5 \%) \\
44(68.8 \%) \\
12(18.8 \%)\end{array}$ & $\begin{array}{c}12(25 \%) \\
30(62.5 \%) \\
6(12.5 \%)\end{array}$ & $\begin{array}{c}33(14.1 \%) \\
136(58.4 \%) \\
64(27.5 \%)\end{array}$ & $0.001 *$ \\
\hline \multicolumn{6}{|l|}{ WAI dimensions } \\
\hline $\begin{array}{l}\text { 1: Current work ability } \\
\text { compared with lifetime best } \\
\text { a }\end{array}$ & $8.51 \pm 2.08$ & $8.62 \pm 1.75$ & $8.29 \pm 1.66$ & $8.50 \pm 1.91$ & 0.656 \\
\hline $\begin{array}{l}\text { 2: Work ability in relation to } \\
\text { work demands } \\
\text { - Physical demands } \\
\text { - Moderate } \\
\text {-Good } \\
\text {-Excellent }\end{array}$ & $\begin{array}{l}20(16.5 \%) \\
43(35.5 \%) \\
58(47.9 \%)\end{array}$ & $\begin{array}{c}6(9.4 \%) \\
32(50 \%) \\
26(40.6 \%)\end{array}$ & $\begin{array}{c}4(8.3 \%) \\
32(66.7 \%) \\
12(25 \%)\end{array}$ & $\begin{array}{c}30(12.9 \%) \\
107(45.9 \%) \\
96(41.2 \%)\end{array}$ & $0.006 *$ \\
\hline
\end{tabular}


Abdelrehim MG et al.

\begin{tabular}{|c|c|c|c|c|c|}
\hline $\begin{array}{l}\text { - Mental demands } \\
\text {-Moderate } \\
\text {-Good } \\
\text {-Excellent }\end{array}$ & $\begin{array}{l}22(18.2 \%) \\
40(33.1 \%) \\
59(48.8 \%)\end{array}$ & $\begin{array}{c}7(10.9 \%) \\
27(42.2 \%) \\
30(46.9 \%)\end{array}$ & $\begin{array}{c}3(6.3 \%) \\
28(58.3 \%) \\
17(35.4 \%)\end{array}$ & $\begin{array}{c}32(13.7 \%) \\
95(40.8 \%) \\
106(45.5 \%)\end{array}$ & $0.028 *$ \\
\hline $\begin{array}{l}\text { 3: Number of diagnosed } \\
\text { diseases a }^{\text {a }}\end{array}$ & $5.08 \pm 1.71$ & $5.41 \pm 1.21$ & $5.85 \pm 1.38$ & $5.57 \pm 1.44$ & $0.004 *$ \\
\hline $\begin{array}{l}\text { 4: Work impairments due to } \\
\text { disease } \\
\text {-No hindrance } \\
\text {-My job causes some } \\
\text { symptoms } \\
\text {-I sometimes slow down work } \\
\text { pace/change work method } \\
\text {-I often slow down/pace/do } \\
\text { only part-time work }\end{array}$ & $\begin{array}{c}83(68.6 \%) \\
27(22.3 \%) \\
4(3.3 \%) \\
7(5.8 \%)\end{array}$ & $\begin{array}{c}36(56.3 \%) \\
21(32.8 \%) \\
5(7.8 \%) \\
2(3.1 \%)\end{array}$ & $\begin{array}{c}18(37.5 \%) \\
23(47.9 \%) \\
3(6.3 \%) \\
4(8.3 \%)\end{array}$ & $\begin{array}{c}137(58.8 \%) \\
71(30.5 \%) \\
12(5.2 \%) \\
13(5.5 \%)\end{array}$ & $0.012 *$ \\
\hline $\begin{array}{l}\text { 5: Sick leave during past year } \\
- \text { None } \\
- \text { At most } 9 \text { days } \\
-10-24 \text { days } \\
-25 \text { days or more }\end{array}$ & $\begin{array}{c}67(55.4 \%) \\
35(28.9 \%) \\
12(9.9 \%) \\
7(5.8 \%)\end{array}$ & $\begin{array}{c}34(53.1 \%) \\
8(12.5 \%) \\
19(29.7 \%) \\
3(4.7 \%)\end{array}$ & $\begin{array}{c}28(58.3 \%) \\
6(12.5 \%) \\
12(25 \%) \\
2(4.2 \%)\end{array}$ & $\begin{array}{c}129(55.4 \%) \\
49(21 \%) \\
43(18.5 \%) \\
12(5.2 \%)\end{array}$ & $0.006 *$ \\
\hline $\begin{array}{l}\text { 6: Own work ability } \\
\text { prognosis for } 2 \text { years } \\
\text { - Hardly able to work } \\
\text {-Not sure } \\
\text {-Fairly sure }\end{array}$ & $\begin{array}{c}6(5 \%) \\
33(27.2 \%) \\
82(67.8 \%) \\
\end{array}$ & $\begin{array}{c}3(4.7 \%) \\
31(48.4 \%) \\
30(46.9 \%) \\
\end{array}$ & $\begin{array}{c}3(6.3 \%) \\
17(35.4 \%) \\
28(58.3 \%) \\
\end{array}$ & $\begin{array}{c}12(5.1 \%) \\
81(34.8 \%) \\
140(60.1 \%) \\
\end{array}$ & 0.071 \\
\hline $\begin{array}{l}\text { 7: Mental resources in the } \\
\text { past month } \\
\text { Enjoyment of daily activities } \\
\text { - Often/ Rather often } \\
\text { - Sometimes } \\
\text {-Rather seldom/Never }\end{array}$ & $\begin{array}{l}58(47.9 \%) \\
42(34.7 \%) \\
21(17.4 \%)\end{array}$ & $\begin{array}{c}24(37.5 \%) \\
32(50 \%) \\
8(12.5 \%)\end{array}$ & $\begin{array}{c}15(31.3 \%) \\
28(58.3 \%) \\
5(10.4 \%)\end{array}$ & $\begin{array}{c}97(41.6 \%) \\
102(43.8 \%) \\
34(14.6 \%)\end{array}$ & $0.046 *$ \\
\hline $\begin{array}{l}\text { Be physical/psychological } \\
\text { active } \\
\text { - Often/ Rather often } \\
\text {-Sometimes } \\
\text {-Rather seldom/Never }\end{array}$ & $\begin{array}{l}63(52.1 \%) \\
42(34.7 \%) \\
16(13.2 \%)\end{array}$ & $\begin{array}{c}28(43.8 \%) \\
33(51.6 \%) \\
3(4.7 \%)\end{array}$ & $\begin{array}{c}21(43.8 \%) \\
25(52.1 \%) \\
2(4.2 \%)\end{array}$ & $\begin{array}{c}112(48.1 \%) \\
100(42.9 \%) \\
21(9 \%)\end{array}$ & $0.045^{*}$ \\
\hline $\begin{array}{l}\text { Optimism about the future } \\
\text { - Often/ Rather often } \\
\text {-Sometimes } \\
\text {-Rather seldom/Never }\end{array}$ & $\begin{array}{c}51(42.1 \%) \\
47(38.8 \%) \\
23(19 \%)\end{array}$ & $\begin{array}{c}23(35.9 \%) \\
32(50 \%) \\
9(14.1 \%)\end{array}$ & $\begin{array}{c}24(50 \%) \\
16(33.3 \%) \\
8(16.7 \%)\end{array}$ & $\begin{array}{l}98(42.1 \%) \\
95(40.8 \%) \\
40(17.2 \%)\end{array}$ & 0.407 \\
\hline
\end{tabular}

${ }^{a}$ Mean \pm SD

*: Statistically significant,
WAI: Work ability index

**: Highly statistically significant. 
Table 3 showed the WAI score of the studied group; it was categorized into poor for $14.1 \%$, suboptimal for $58.4 \%$ and optimal for $27.5 \%$. Regarding the dimensions of work ability, there were significant differences between the age categories in all dimensions except the current work ability compared with lifetime best and work ability prognosis for 2 years. The old age group ( $\geq 40$ years) had a lower percentage of optimal WAI and was less likely to have a better response in most dimensions of WAI.

The mean WAI of the study subjects was $40.6( \pm 4.6)$ and the range was 23-49. The current work ability compared with lifetime best was $8.5 \pm 1.91$ (Results were not tabulated).

Table 4: Bivariate associations of socio-demographics, chronic diseases and work characteristics with perceived work ability.

\begin{tabular}{|c|c|c|c|}
\hline & \multicolumn{2}{|c|}{ Perceived work ability ${ }^{a}$} & \multirow[b]{2}{*}{ p-value } \\
\hline & $\begin{array}{c}\text { Poor } \\
\text { Crude OR }(95 \% \mathrm{CI}) \\
\end{array}$ & $\begin{array}{c}\text { Optimal } \\
\text { Crude OR }(95 \% \mathrm{CI}) \\
\end{array}$ & \\
\hline \multicolumn{4}{|c|}{ Socio-demographics } \\
\hline \multicolumn{4}{|c|}{ Age (year) } \\
\hline $20-29$ & 1 & 1 & \\
\hline $30-39$ & $0.87(0.33-2.27)$ & $0.37(0.17-0.77)$ & \\
\hline$\geq 40$ & $1.91(0.78-4.68)$ & $0.27(0.10-0.70)$ & 0.001* \\
\hline \multicolumn{4}{|l|}{ Sex } \\
\hline Female & 1 & 1 & \\
\hline Male & $0.48(0.17-1.33)$ & $1.50(1.10-2.93)$ & $0.045 *$ \\
\hline \multicolumn{4}{|c|}{ Marital status } \\
\hline Unmarried & 1 & 1 & \\
\hline Married & $0.92(0.41-2.07)$ & $0.52(0.28-0.96)$ & 0.109 \\
\hline \multicolumn{4}{|l|}{ Residence } \\
\hline Urban & 1 & 1 & \\
\hline Rural & $1.18(0.55-2.53)$ & $1.18(0.65-2.17)$ & 0.821 \\
\hline \multicolumn{4}{|l|}{ Smoking } \\
\hline Non-smoker & 1 & 1 & \\
\hline Smoker & $0.34(0.10-1.21)$ & $0.65(0.24-1.77)$ & \\
\hline Ex-smoker & $0.80(0.25-2.60)$ & $0.62(0.28-1.37)$ & 0.342 \\
\hline \multicolumn{4}{|l|}{ BMI $^{\text {b }}$} \\
\hline $18.5-24.9$ & 1 & 1 & \\
\hline $25-29.9$ & $0.77(0.30-1.98)$ & $0.60(0.30-1.18)$ & \\
\hline$\geq 30$ & $1.12(0.44-2.81)$ & $0.36(0.16-0.82)$ & 0.093 \\
\hline
\end{tabular}




\begin{tabular}{|c|c|c|c|}
\hline \multicolumn{4}{|c|}{ Reproductive history for females $(\mathrm{No}=168)$} \\
\hline Number of children & $0.91(0.67-1.24)$ & $0.65(0.48-1.89)$ & 0.061 \\
\hline $\begin{array}{l}\text { Breast feeding } \\
\text { NO } \\
\text { Yes }\end{array}$ & $0.45(0.12-1.62)$ & $0.64(0.24-1.71)$ & 0.344 \\
\hline $\begin{array}{l}\text { Hormonal contraception } \\
\text { NO } \\
\text { Yes }\end{array}$ & $1.12(0.44-2.86)$ & $0.79(0.33-1.87)$ & 0.800 \\
\hline $\begin{array}{l}\text { Work characteristics } \\
\text { Work category }\end{array}$ & & & \\
\hline $\begin{array}{l}\text { Nurses } \\
\text { Physicians } \\
\text { Technicians }\end{array}$ & $\begin{array}{c}1 \\
3.12(1.30-7.50) \\
1.33(0.47-3.81)\end{array}$ & $\begin{array}{c}1 \\
0.57(0.24-1.37) \\
0.60(0.26-1.36)\end{array}$ & $0.022 *$ \\
\hline $\begin{array}{l}\text { Night shift } \\
\mathrm{NO} \\
\text { Yes }\end{array}$ & $1.12(0.52-2.41)$ & $0.81(0.44-1.48)$ & 0.702 \\
\hline $\begin{array}{l}\text { Specialty } \\
\text { Anesthesia, surgery and obstetrics } \\
\text { Pediatric and internal medicine } \\
\text { Laboratory technicians }\end{array}$ & $\begin{array}{c}1 \\
1.45(0.62-3.42) \\
1.15(0.38-3.46)\end{array}$ & $\begin{array}{c}1 \\
1.11(0.58-2.13) \\
0.73(0.30-1.78)\end{array}$ & 0.815 \\
\hline Working hours/day & $1.02(0.84-1.24)$ & $1.08(0.94-1.24)$ & 0.576 \\
\hline Distance to reach work & $1.01(0.99-1.03)$ & $1.01(1.00-1.03)$ & 0.141 \\
\hline History of chronic diseases & & & \\
\hline $\begin{array}{l}\text { Musculoskeletal } \\
\text { NO } \\
\text { Yes }\end{array}$ & $1.83(0.84-4.01)$ & $0.35(0.15-0.81)$ & $0.003 *$ \\
\hline $\begin{array}{l}\text { Cardiovascular } \\
\mathrm{NO} \\
\text { Yes }\end{array}$ & $0.72(0.29-1.80)$ & $0.10(0.02-0.37)$ & $<0.0001 * *$ \\
\hline $\begin{array}{l}\text { Respiratory } \\
\text { NO } \\
\text { Yes }\end{array}$ & $1.65(0.76-3.60)$ & $0.32(0.14-0.73)$ & $0.002 *$ \\
\hline $\begin{array}{l}\text { Gastrointestinal } \\
\text { NO } \\
\text { Yes }\end{array}$ & $1.75(0.75-4.12)$ & $0.13(0.03-0.57)$ & $<0.0001 * *$ \\
\hline $\begin{array}{l}\text { Mental } \\
\mathrm{NO} \\
\mathrm{Yes}\end{array}$ & $\begin{array}{c}1 \\
1.84(0.60-5.66)\end{array}$ & $\begin{array}{c}1 \\
1.18(0.84-5.01)\end{array}$ & 0.201 \\
\hline
\end{tabular}

a Multinomial Logistic Regression and the reference category was suboptimal work ability b BMI: Body Mass Index *: Statistically significant, **: Highly statistically significant. 
Table 4 showed the bivariate associations between WAI categories and characteristics of the studied population. Age, sex, work category, musculoskeletal, cardiovascular, respiratory, and gastrointestinal diseases were significantly associated with WAI level.

Table 5: Determinants of perceived work ability among the studied healthcare providers.

\begin{tabular}{|c|c|c|c|}
\hline & \multicolumn{2}{|c|}{ Perceived work ability ${ }^{\text {a }}$} & \multirow[b]{2}{*}{ p-value } \\
\hline & $\begin{array}{c}\text { Poor } \\
\text { Adjusted OR }(95 \% \mathrm{CI})\end{array}$ & $\begin{array}{c}\text { Optimal } \\
\text { Adjusted OR (95\%CI) } \\
\end{array}$ & \\
\hline \multicolumn{4}{|l|}{ Age (years) } \\
\hline $20-29$ & 1 & 1 & \\
\hline $30-39$ & $0.62(0.21-1.79)$ & $0.68(0.29-1.59)$ & \\
\hline$\geq 40$ & $1.77(0.67-4.72)$ & $0.33(0.11-0.96)$ & $0.041 *$ \\
\hline \multicolumn{4}{|l|}{ Sex } \\
\hline Female & 1 & 1 & \\
\hline Male & $0.45(0.15-1.37)$ & $2.38(1.10-5.22)$ & $0.017 *$ \\
\hline \multicolumn{4}{|l|}{ Work category } \\
\hline Nurses & 1 & 1 & \\
\hline Physicians & $3.61(1.39-9.34)$ & $0.56(0.21-1.49)$ & \\
\hline Technicians & $1.27(0.42-3.87)$ & $0.43(0.16-1.11)$ & $0.013 *$ \\
\hline \multicolumn{4}{|l|}{ Musculoskeletal } \\
\hline NO & 1 & 1 & \\
\hline Yes & $1.79(1.01-10.44)$ & $0.35(0.14-0.87)$ & $0.014 *$ \\
\hline \multicolumn{4}{|l|}{ Cardiovascular } \\
\hline Yes & $1.05(0.36-3.01)$ & $0.06(0.01-0.27)$ & $<0.0001 * *$ \\
\hline \multicolumn{4}{|l|}{ Respiratory } \\
\hline $\mathrm{NO}$ & 1 & 1 & \\
\hline Yes & $1.52(0.66-3.52)$ & $0.32(0.13-0.79)$ & $0.013 *$ \\
\hline \multicolumn{4}{|l|}{ Gastrointestinal } \\
\hline Yes & $1.35(0.54-3.39)$ & $0.15(0.03-0.71)$ & $0.009 *$ \\
\hline
\end{tabular}

a Multinomial Logistic Regression and the reference category was the suboptimal work ability *: Statistically significant, **: Highly statistically significant.

Table 5 showed the final multivariable regression analysis which demonstrated that age, sex, work category and chronic diseases were the significant predictors of perceived work ability among the studied healthcare providers. The adjusted ORs for optimal work ability (reference is suboptimal WAI) for old subjects versus the youngest was 0.33 (95\% CI, 0.11-0.96), for males versus females was 2.38 (95\% CI, $1.10-5.22)$ and for physicians versus nurses was 0.56 (95\% CI, 0.21-1.49). 


\section{Discussion}

Demographic changes and population aging around the world including the Arab countries imply increasing the average age of the labor force and workers with health problems which can lead to decreased work ability (Kühn et al., 2018). The healthcare providers are exposed to high levels of physical, mental and emotional stressors that can influence their performance and efficiency and have a negative impact on their work ability and health-related quality of life (Costa et al., 2005; Koinis et al., 2015; Mehrdad et al., 2016). Consequently, efforts to assess, maintain, and promote the ability to work on current and future healthcare providers are of key importance.

The results of the current study identified that the mean WAI score of the studied subjects was $40.6( \pm 4.6)$ (Results were not tabulated) which approximates that previously recorded value among the healthcare personnel in Iran (40.3 \pm 5.2 ) (Mehrdad et al., 2016) and in Italy (39.7 \pm 5.4$)$ (Costa et al., 2005). The study categorized the participants' work ability based on their WAI score into poor $(14.1 \%)$, suboptimal $(58.4 \%)$ and optimal $(27.5 \%)$ work ability (Table 3$)$. These percentages were similar to the previous values reported in Italy (Costa et al., 2005), however poor/moderate
WAI had a higher percentage (23.3\%) .

The present study showed that the significant predictors of WAI among the healthcare providers in Minia city were age, sex, work category and chronic disease conditions (including musculoskeletal, cardiovascular, respiratory and gastrointestinal diseases) (Table 5).

One of the most studied demographic factors that influence the work ability was the age. The current work found that the old age group ( $\geq 40$ years) was less likely to have a better response in most dimensions of WAI (Table 3). Older healthcare providers had lower odds for optimal WAI which support the previous findings of most studies that reported a decreased WAI with older age (Monteiro et al., 2006; Golubic et al., 2009; El Fassi et al., 2013; Mehrdad et al., 2016; Heyam et al., 2018), while other studies demonstrated no association (Juszczyk et al., 2019 and Pranjic et al., 2019). With old age, the physical and mental skills will be reduced due to suffering from various health problems and chronic diseases which can limit the ability to work (Converso et al., 2018). In the older age groups, infirmity, inability to cope, and job strain are more common (Gould et al., 2008). Inconsistent relations between age and work ability 
may be due to age homogeneity within the samples of different studies. The age 40 is still relatively young and there may be a heterogenic effect of age among younger old and older old workers. Moreover, aging workers accumulate more job and personal resources as tenure and experience, which may buffer the negative effects of the aging process on their perceptions of work ability $(\mathrm{Ng}$ and Feldman, 2013).

In the current study, males have higher ORs for optimal work ability versus females $(2.38,95 \%$ CI: 1.10 5.22) (Table5); that consisted with the research carried out among healthcare personnel and concluded that women had significantly lower WAI than men (Costa et al., 2005; Costa and Sartori, 2007; Mehrdad et al., 2016). While, in research among healthcare workers in the UK, WAI was reported to be higher among women (Nachiappan and Harrison, 2005). Sex differences in work ability might be related to the working conditions, it was proved that jobs implying high physical and mental constraints may have a stronger impact on work ability, particularly among women (Costa and Sartori, 2007). Also, it was found that female physicians utilize emotional coping strategies for the stressful situations at work, such as wishful thinking and seeking help from God, while male physicians employ more often problem solving and positive approaches to deal with difficult situations which increase their coping with workloads and perceived work ability (Koinis et al., 2015).

Other studies detected that sex had no effect on work ability like what were done by Monteiro et al., 2006 on the work ability of workers in different age groups in a public health institution in Brazil and Pranjic et al., 2019 in their work on perceived work ability index of public service employees in relation to ageing and gender.

In the present work, the results showed that the marital status had no effect on the work ability (Table 4). On the contrary, being divorced or widowed was considered by Pranjic et al., 2019 as a predictor of poor WAI. Indeed, less was known about the impact of the marital state on WAI and further research is needed to address this issue.

The present work detected that physicians were more likely to have poor WAI than nurses and technicians which was similar with previous study done by Nachiappan and Harrison, 2005 in United Kindom. However, Mehrdad et al., 2016 in their study on work ability index among healthcare personnel in a 
university hospital in Tehran, Iran; found a better work ability for physicians in comparison to nurses and technicians. Lower work ability among physicians in the current study might be due to the higher physical and mental demands of work in the existence of deficient resources (Gharibi et al., 2016 and Pranjic et al., 2019), and more work responsibilities with higher exposure to stress at work. Previously, Gharibi et al., 2016 found significant correlations between WAI and work-related stress. Moreover, high physical work demands have been associated with low work ability and long-term sick leave among heterogeneous study populations (van den Berg et al., 2009; Leijten et al., 2013; van den Berg et al., 2017).

In accordance with previous researches (Costa et al., 2005; El Fassi et al., 2013; Leijten et al., 2014; van den Berg et al., 2017), healthcare providers with chronic diseases were more likely to have low work ability and long-term sick leave than healthy individuals. The current study supported the negative impact of musculoskeletal, cardiovascular, respiratory, and gastrointestinal disorders on having optimal work ability (Table 5). While Costa et al., 2005 reported that skin and neuropsychic illnesses appear to lower
WAI to a greater extent than other health disorders as musculoskeletal, respiratory and cardiac.

Musculoskeletal disorders have a profound impact on individual health, regular work activities, sickness absence, and early retirement, particularly in physically demanding occupations (Leijten et al., 2014). Senior workers may have particular difficulties in coping with physically demanding occupations while maintaining good health (Lunde et al., 2014). A previous study confirmed that multi-site musculoskeletal pain had an association with poor work ability among health care providers. The magnitude of association was likely to increase by a higher number of pain sites (Phongamwong and Deema, 2015). Moreover, musculoskeletal, cardiovascular, mental and respiratory diseases have been identified as important causes of sick leave and were predictive of sustained suboptimal health status over the subsequent years which can explain their association with low work ability (van den Berg et al., 2017).

Concerning lifestyle-related factors, like smoking and obesity, they were not significantly associated with perceived work ability in the current work (Table 4) which is supported by previous studies (Kaleta et al., 2006; van den Berg et 
al., 2009; Mehrdad et al., 2016). While contradictory findings have reported the relation between smoking, obesity and work ability (Robroek et al., 2011 and El Fassi et al., 2013). van den Berg et al, 2017 reported less consistent associations between heavy smoking and work ability that differed across disease subgroups of the participants. Also, obesity was associated with low work ability, only among employees with respiratory disease. Their findings were explained that smoking and obesity contribute to the severity of respiratory and cardiovascular symptoms, therefore, has an important impact on work ability and sick leave in these particular subgroups (van den Berg et al., 2017).

\section{Strengths and limitations}

The strength of this study implies the study population which consisted of paid healthcare providers in a developing country. To our knowledge, this is the first study about the work ability that was carried out among this occupational group at Minia. Moreover, the study focused on various related variables and included all age groups, both males and females, and different work categories within the healthcare sector to be able to examine the effects of these variables on the perceived work ability. Some limitations of this study should be mentioned: first, inherent to the crosssectional design of the study, causality between the determinants and work ability cannot be assessed. Second, the data were self-reported; so, there may be a potential for response bias. However, self-reported data were frequently used in research of occupational medicine and it had proved to be a good representative for the association between low work ability and common diseases (van den Berg et al., 2017). Finally, the study used broad categories of common diseases and severity of the condition was not assessed. For future research, it is recommended to have more insight into specific diagnoses and the association between the severity of diseases and work impairments.

Conclusion : the current study supports the evidence that age, sex, work category and chronic disease conditions can significantly affect the work ability of healthcare providers. Therefore, it is important to adopt strategies for health promotion, prevention and control of chronic diseases particularly in old age to maintain and improve the work ability of this occupational group.

\section{Conflict of interest}

All authors have no conflict of interest to declare. 


\section{Funding}

\section{None}

\section{Acknowledgment}

The authors are grateful to all healthcare providers who participated in the study or facilitated the data collection by the investigators for their kind cooperation.

\section{References}

1. Converso D, Sottimano I, Guidetti G, Loera B, Cortini M, et al. (2018): Aging and Work Ability: The Moderating Role of Job and Personal Resources. Front Psychol; 8:2262. DOI: $10.3389 /$ fpsyg.2017.02262

2. Costa G, Sartori S, Bertoldo B, Olivato D, Antonacci G, et al. (2005): Work ability in health care workers. International Congress Series; 1280:264-9.DOI:https://doi.org/10.1016/j. ics.2005.02.079

3.Costa G and Sartori S (2007): Ageing, working hours and work ability. Ergonomics; 50(11):1914-30

4. de Zwart BC, Frings-Dresen $\mathrm{MH}$, and van Duivenbooden JC (2002): Test-retest reliability of the Work Ability Index questionnaire. Occup Med (Lond); 52(4):177-81. DOI:10.1093/ occmed/52.4.177

5. El Fassi M, Bocquet V, Majery N, Lair ML, Couffignal S, et al. (2013): Work ability assessment in a worker population: comparison and determinants of Work Ability Index and Work Ability score. BMC Public Health; 13(1):305

6. Elden N, Rizk H, and Wahby G (2016): Improving health system in Egypt: Perspectives of physicians. Egyptian Journal of Community Medicine; 34(1):45-58

7. Gharibi V, Mokarami H, Taban A, Aval MY, Samimi K, et al. (2016): Effects of work-related stress on work ability index among Iranian workers. Safety and health at work; 7(1):43-8

8. Golubic R, Milosevic M, Knezevic B, and Mustajbegovic J (2009): Work $\square$ related stress, education and work ability among hospital nurses. J Adv Nurs; 65(10):2056-66

9. Gould R, Ilmarinen J, Järvisalo J, and Koskinen S (2008): Dimensions of work ability: results of the Health 2000 Survey. Finnish Institute of Occupational Health, Helsinki https:// www.julkari.fi/bitstream/handle/10024/78055/ dimensions_of_work_ability_7.pdf

10. Guo Y, Reeuwijk KG, Robroek SJW, Niessen MAJ, Kraaijenhagen RA, et al. (2015): The Prognostic Value of the Work Ability Index for Sickness Absence among Office Workers. PLoS One; 10(5):e0126969 . DOI:10.1371/journal. pone. 0126969

11. Heyam DF, Besher G, and Nesreen AK (2018): Work Ability Index of Shift Working Hospital Nurses in Jordan. Open Nurs J; 12:116-24 . DOI:10.2174/1874434601812010116

12. Juszczyk G, Czerw AI, Religioni U, Olejniczak D, Walusiak-Skorupa J, et al. (2019): Work Ability Index (WAI) values in a sample of the working population in Poland. Ann Agric Environ Med; 26(1):78-84 . DOI:10.26444/aaem/91471

13. Kaleta D, Makowiec-Dąbrowska T, and Jegier A (2006): Lifestyle index and work ability. Int J Occup Med Environ Health; 19(3):170-7

14. Koinis A, Giannou V, Drantaki V, Angelaina S, Stratou E, et al.(2015): The Impact of Healthcare Workers Job Environment on Their Mentalemotional Health. Coping Strategies: The Case of a Local General Hospital. Health Psychol Res; 3(1):1984. DOI:10.4081/hpr.2015.1984

15. Kühn S, Milasi S, and Yoon S (2018): Population ageing and future labour market challenges. World Employment and Social Outlook; 2018(1):45-50

16. Leijten FR, van den Heuvel SG, Ybema JF, Robroek SJ, and Burdorf A (2013): Do work factors modify the association between chronic health problems and sickness absence among 
older employees? Scand J Work Environ Health; 39(5):477-85 . DOI:10.5271/sjweh.3353

17. Leijten FR, van den Heuvel SG, Ybema JF, van der Beek AJ, Robroek SJ, et al. (2014): The influence of chronic health problems on work ability and productivity at work: a longitudinal study among older employees. Scand J Work Environ Health:473-82

18. Lunde LK, Koch M, Knardahl S, Waersted M, Mathiassen SE, et al. (2014): Musculoskeletal health and work ability in physically demanding occupations: study protocol for a prospective field study on construction and health care workers. BMC Public Health; 14:1075. DOI:10.1186/1471-2458-14-1075

19. Mehrdad R, Mazloumi A, Arshi S, and Kazemi $Z$ (2016): Work ability index among healthcare personnel in a university hospital in Tehran, Iran. Work (Reading, Mass); 53(4):851-7. DOI:10.3233/WOR-162277

20. Monteiro MS, Ilmarinen J, and Filho HRC (2006): Work ability of workers in different age groups in a public health institution in Brazil. Int J Occup Saf Ergon; 12(4):417-27

21. Nachiappan N and Harrison J (2005): Work ability among health care workers in the United Kingdom: A pilot. International Congress Series; 1280:286-91. DOI:https://doi. org/10.1016/j.ics.2005.02.082

22. Ng TW and Feldman DC (2013): A metaanalysis of the relationships of age and tenure with innovation-related behaviour. J Occup Organ Psychol; 86(4):585-616 doi:10.1111/ joop.12031

23. Palmlof L, Skillgate E, Talback M, Josephson M, Vingard E, et al. (2019): Poor work ability increases sickness absence over 10 years. Occup Med (Lond); 69(5):359-65. DOI:10.1093/ occmed/kqz083

24. Phongamwong $C$ and Deema $H$ (2015): The impact of multi-site musculoskeletal pain on work ability among health care providers. J Occup Med Toxicol; 10:21 . DOI:10.1186/ s12995-015-0063-8

25. Pranjic N, Gonzales JMG, and CvejanovKezunović L (2019): Perceived work ability index of public service employees in relation to ageing and gender: A comparison in three European countries. Slovanian Journal of Public Health;58(4):179. DOI:https://doi. org/10.2478/sjph-2019-0023

26. Robroek SJW, van den Berg TIJ, Plat JF, and Burdorf A (2011): The role of obesity and lifestyle behaviours in a productive workforce. Occup Environ Med; 68(2):134-9. DOI:10.1136/oem.2010.055962

27. Tuomi K, Ilmarinen J, Jahkola AK and Tulkki A (1998): Work Ability Index. 2nd Edition, Finnish Institute of Occupational Health, Helsinki. Available at: https://fhvmetodik.se/ wp-content/uploads/2013/04/WAIpoang.pdf

28. Tuomi K, Ilmarinen J, Seitsamo J, Huuhtanen P, Martikainen R, et al. (1997): Summary of the Finnish research project (1981-1992) to promote the health and work ability of aging workers. Scand J Work Environ Health; 23 Suppl 1:66-71

29. van den Berg S, Burdorf A, and Robroek SJW (2017): Associations between common diseases and work ability and sick leave among health care workers. Int Arch Occup Environ Health; 90(7):685-93 . DOI:10.1007/s00420-017-12311

30. van den Berg TI, Elders LA, de Zwart BC, and Burdorf A (2009): The effects of work-related and individual factors on the Work Ability Index: a systematic review. Occup Environ Med; 66(4):211-20 . DOI:10.1136/oem.2008.039883 
